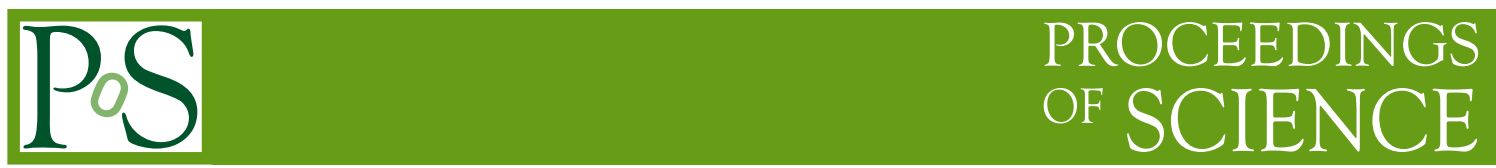

\title{
Charm Leptonic and semi-Leptonic decays at BESIII
}

\author{
Jiangchuan Chen ${ }^{* \dagger}$ \\ (On behalf of the BESIII Collaboration) \\ Institute of High Energy Physics, CAS, Beijing, China, 100049 \\ E-mail: chenjceihep.ac.cn
}

We present recent BESIII results about the study of mesons which contain at least one charm quark. We determined the $D_{(s)}^{+}$decay constants, the form factors of $D$ semi-leptonic decays, the CKM matrix elements $\left|V_{c s(d)}\right|$.

ICHEP2018, XXXIX International Conference on High Energy Physics

4-11 July 2018

Seoul, Korea

* Speaker.

$\dagger$ Work supported in part by the National Natural Science Foundation of China (NSFC) under Contract No. 11675200 


\section{Introduction}

The study of mesons which contain at least one charm quark is referred to as open charm physics. It offers the possibility to study up-type quark transitions. Since the $c$ quark cannot be treated in any mass limit, theoretical predictions are difficult and experimental input is crucial. The at-threshold decay topology offers special opportunities to study open charm decays.

The BESIII is a magnetic spectrometer working at a double-ring $e^{+} e^{-}$collider operating at center-of-mass energy between $2.0 \mathrm{GeV}$ and $4.6 \mathrm{GeV}$, located at the Institute of High Energy Physics (IHEP) in Beijing. The maximum luminosity of the BEPCII at $3.773 \mathrm{GeV}$ is $1 \times$ $10^{33} \mathrm{~cm}^{-2} \mathrm{~s}^{-1}$ [1]. The samples of interest for the analysis described in the following were taken at the $D \bar{D}$ threshold $(3.773 \mathrm{GeV})$ and $D_{s}^{*} D_{s}$ threshold $(4.178 \mathrm{GeV})$ with integrated luminosities of $2.92 \mathrm{fb}^{-1}$ and $3.19 \mathrm{fb}^{-1}$, respectively. Throughout in the following, charge conjugate is implied.

\section{Pure leptonic $D_{(s)}$ decays}

The pure leptonic decay of charged $D_{(s)}^{+}$mesons proceeds via the annihilation of the $c$ quark and the anti- $d$ (anti-s) quark to a virtual $W^{ \pm}$boson and its decay to $\ell^{+} v_{\ell}$. The decay rate can be parameterized as:

$$
\Gamma\left(D_{(s)}^{+} \rightarrow \ell^{+} v_{\ell}\right)=\frac{G_{F}^{2}}{8 \pi} f_{D_{(s)}}^{2} m_{\ell}^{2} m_{D_{(s)}}\left(1-\frac{m_{\ell}^{2}}{m_{D_{(s)}}^{2}}\right)^{2}\left|V_{c d(s)}\right|^{2},
$$

where $G_{F}$ is the Fermi coupling constant, $m_{\ell}$ and $m_{D_{(s)}}$ are the masses of the lepton and the $D_{(s)}$ meson in the final state, respectively.

2.1 $D^{+} \rightarrow \mu^{+} v_{\mu}$

By analyzing the data sample accumulated at $3.773 \mathrm{GeV}$, to select the leptonic $D^{+}$decay with a missing neutrino, we calculated $U_{\text {miss }}=E_{\text {miss }}-p_{\text {miss }}$ or $M_{\text {miss }}^{2}=E_{\text {miss }}^{2}-p_{\text {miss }}^{2}$, where $E_{\text {miss }}$ and $p_{\text {miss }}$ are the missing energy and missing momentum of the event. After subtracting the backgrounds, $409.0 \pm 21.2$ signal events are retained and the branching fraction (BF) is measured to be $B F\left(D^{+} \rightarrow \mu^{+} v_{\mu}\right)=\left(3.71 \pm 0.19_{\text {stat }} \pm 0.06_{\text {syst }}\right) \times 10^{-4}$, which is the most precise measurement to date. Combining this BF measurement and the Particle Data Group (PDG) [2] values of $D^{+}$ lifetime, $m_{D^{+}}, m_{\mu}$ and magnitude of $\left|V_{c d}\right|$ determined from the global Standard Model (SM) fit, the decay constant is determined to be $f_{D}=\left(203.2 \pm 5.3_{\text {stat }} \pm 1.8_{\text {syst }}\right) \mathrm{MeV}$. Alternatively, the magnitude of CKM matrix element $\left|\mathrm{V}_{c d}\right|$ is extracted to be $\left|\mathrm{V}_{c d}\right|=0.2210 \pm 0.0058_{\text {stat }} \pm 0.0047_{\text {syst }}$ [3].

\section{$2.2 D_{s}^{+} \rightarrow \mu^{+} v_{\mu}$}

By analyzing the data taken at $4.178 \mathrm{GeV}$, we also studied the leptonic $D_{s}^{+}$decays. From $14 D_{s}^{-}$hadronic decay modes, preliminarily, $388660 \pm 2592 \mathrm{ST} D_{s}^{-}$mesons were accumulated. In the system recoiling against the ST $D_{s}^{-}$, the signal events of $D_{s}^{+} \rightarrow \mu^{+} v_{\mu}$ decay were selected. We obtained $1135.9 \pm 33.1 D_{s}^{+} \rightarrow \mu^{+} v_{\mu}$, and the preliminary result of absolute BF is determined to be $B F\left(D_{s}^{+} \rightarrow \mu^{+} v_{\mu}\right)=\left(0.550 \pm 0.016_{\text {stat }} \pm 0.015_{\text {syst }}\right) \%$, the decay constant $f_{D_{s}}=$ $245.9 \pm 3.6_{\text {stat }} \pm 3.5_{\text {syst }} \mathrm{MeV}$ and $\left|V_{c s}\right|=0.987 \pm 0.014_{\text {stat }} \pm 0.015_{\text {syst }}$. The additional systematic uncertainties according to the input parameters are negligible for $\left|\mathrm{V}_{c s}\right|$ and 0.3 for $f_{D_{s}}$. 


\section{Semi-leptonic $D_{(s)}$ decays}

\section{1 $D^{0(+)} \rightarrow P e^{+} v_{e}\left(P=K^{-}, \pi^{-}, K^{0}, \pi^{0}\right)$}

In the SM, neglecting the lepton mass, the differential decay rate for $D^{0(+)} \rightarrow P e^{+} v_{e}(P=$ $K^{-}, \pi^{-}, K^{0}$ or $\pi^{0}$ ) is given by

$$
\frac{d \Gamma}{d q^{2}}=X \frac{G_{F}^{2}}{24 \pi^{3}}\left|V_{\mathrm{cd}(\mathrm{s})}\right|^{2} p^{3}\left|f_{+}^{P}\left(q^{2}\right)\right|^{2},
$$

where $X$ is a multiplicative factor due to isospin, which equals to 1 for modes $D^{0} \rightarrow K^{-} e^{+} v_{e}$, $D^{0} \rightarrow \pi^{-} e^{+} v_{e}, D^{+} \rightarrow K^{0} e^{+} v_{e}$, and $1 / 2$ for mode $D^{+} \rightarrow \pi^{0} e^{+} v_{e}, p$ is the momentum of the pseudoscalar meson $P$ in the rest frame of the $D$ meson, $q^{2}$ is the squared four momentum transfer, i.e., the invariant mass of the electron and neutrino system, $f_{+}^{P}\left(q^{2}\right)$ is the form factor which describes the strong interaction between the final state quarks and is usually parameterized in data analysis.

Based on the data taken at $3.773 \mathrm{GeV}$, BESIII studies the dynamics of the $D^{0} \rightarrow K^{-} e^{+} v_{e}$ and $D^{0} \rightarrow \pi^{-} e^{+} v_{e}$ decays. The BFs are measured to be $B F\left(D^{0} \rightarrow K^{-} e^{+} v_{e}\right)=\left(3.505 \pm 0.014_{\text {stat }} \pm\right.$ $\left.0.033_{\text {syst }}\right) \%$ and $B F\left(D^{0} \rightarrow \pi^{-} e^{+} v_{e}\right)=\left(0.295 \pm 0.004_{\text {stat }} \pm 0.003_{\text {syst }}\right) \%$ [5]. Similarly, the BFs are measured to be $B F\left(D^{+} \rightarrow \bar{K}^{0} e^{+} v_{e}\right)=\left(8.604 \pm 0.056_{\text {stat }} \pm 0.151_{\text {syst }}\right) \%$ and $B F\left(D^{+} \rightarrow \pi^{0} e^{+} v_{e}\right)=$ $\left(0.363 \pm 0.008_{\text {stat }} \pm 0.005_{\text {syst }}\right) \%$ [6].

We also studied the differential decay rates of these two processes. We extract the product $f_{+}(0)\left|V_{c s(d)}\right|$ and other form factor parameters. Using the values for $f_{+}^{K(\pi)}(0)\left|V_{c s(d)}\right|$ from the twoparameter $z$-series expansion fits and with $f_{+}^{K}(0)=0.747 \pm 0.011 \pm 0.015[7]$ and $f_{+}^{\pi}(0)=0.666 \pm$ $0.020 \pm 0.021$ [8] calculated in LQCD, $\left|V_{c S}\right|$ is obtained to be $0.9601 \pm 0.0033 \pm 0.0047 \pm 0.0239$ $\left(\left|V_{c d}\right|=0.2155 \pm 0.0027 \pm 0.0014 \pm 0.0094\right)$, where the first uncertainties are statistical, the second ones systematic, and the third ones are due to the theoretical uncertainties in the form factor calculations. The BESIII results are in good agreement with the previous measurements, and with the best precision to date.

\section{$3.2 D^{0(+)} \rightarrow P \mu^{+} v_{\mu}\left(P=K^{-}, \pi^{-}, K^{0}, \pi^{0}\right)$}

Based on the data sample collected at $\sqrt{s}=3.773 \mathrm{GeV}$, BESIII although studied $D^{0(+)} \rightarrow$ $P \mu^{+} v_{\mu}\left(P=K^{-}, \pi^{-}, K^{0}, \pi^{0}\right)$. The BFs and the lepton universality ratios $R_{L U}=B F\left(D^{0(+)} \rightarrow\right.$ $\left.P \mu^{+} v_{\mu}\right) / B F\left(D^{0(+)} \rightarrow P e^{+} v_{e}\right)$ are measured to be $B F\left(D^{0} \rightarrow K^{-} \mu^{+} v_{\mu}\right)=\left(3.429 \pm 0.019_{\text {stat }} \pm\right.$ $\left.0.035_{\text {syst }}\right) \%\left(R_{L U}=0.978 \pm 0.007_{\text {stat }} \pm 0.012_{\text {syst }}\right)[9], B F\left(D^{+} \rightarrow \bar{K}^{0} \mu^{+} v_{\mu}\right)=\left(8.72 \pm 0.07_{\text {stat }} \pm\right.$ $\left.0.18_{\text {syst }}\right) \%\left(R_{L U}=0.988 \pm 0.033\right)[10], B F\left(D^{0} \rightarrow \pi^{-} \mu^{+} v_{\mu}\right)=\left(0.267 \pm 0.007_{\text {stat }} \pm 0.007_{\text {syst }}\right) \%$ $\left(R_{L U}=0.905 \pm 0.027_{\text {stat }} \pm 0.023_{\text {syst }}\right)$ and $B F\left(D^{+} \rightarrow \pi^{0} \mu^{+} v_{\mu}\right)=\left(0.342 \pm 0.011_{\text {stat }} \pm 0.010_{\text {syst }}\right) \%$ $\left(R_{L U}=0.942 \pm 0.037_{\text {stat }} \pm 0.027_{\text {syst }}\right)[11]$.

\section{3 $D_{s}^{+} \rightarrow K^{(*) 0} e^{+} v_{e}$}

Using the data sample collected at $\sqrt{s}=4.178 \mathrm{GeV}$, BESIII measured $D_{s}^{+} \rightarrow K^{0} e^{+} v_{e}$ and $D_{s}^{+} \rightarrow K^{* 0} e^{+} v_{e}$. The BFs are obtained to be $B F\left(D_{s}^{+} \rightarrow K^{0} e^{+} v_{e}\right)=\left(3.25 \pm 0.38_{\text {stat }} \pm 0.16_{\text {syst }}\right) \%$ and $B F\left(D_{s}^{+} \rightarrow K^{* 0} e^{+} v_{e}\right)=\left(2.37 \pm 0.26_{\text {stat }} \pm 0.20_{\text {syst }}\right) \%$ [12]. The first measurements of the hadronic form-factor parameters are obtained. The result for $D_{s}^{+} \rightarrow K^{0} e^{+} v_{e}$ is $f_{+}(0)=0.720 \pm 0.084$ (stat) \pm 0.013 (syst), and for $D_{s}^{+} \rightarrow K^{* 0} e^{+} v_{e}$ decay, the form-factor ratios are $r_{V}=V(0) / A_{1}(0)=1.67 \pm$ 0.34 (stat) \pm 0.016 (syst) and $r_{2}=A_{2}(0) / A_{1}(0)=0.77 \pm 0.28($ stat $) \pm 0.07$ (syst). 


\section{$3.4 D_{s}^{+} \rightarrow \eta\left(\eta^{\prime}\right) e^{+} v_{e}$}

By analyzing the data taken at $4.178 \mathrm{GeV}$, BESIII measured the absolute BFs for semi-leptonic $D_{s}^{+} \rightarrow \eta\left(\eta^{\prime}\right) e^{+} v_{e}$ decays. The preliminary results are $B F\left(D_{s}^{+} \rightarrow \eta e^{+} v_{e}\right)=\left(2.32 \pm 0.06_{\text {stat }} \pm\right.$ $\left.0.06_{\text {syst }}\right) \%$ and $B F\left(D_{s}^{+} \rightarrow \eta^{\prime} e^{+} v_{e}\right)=\left(0.82 \pm 0.07_{\text {stat }} \pm 0.03_{\text {syst }}\right) \%$, and combining the BFs of $B F_{D^{+} \rightarrow \eta\left(\eta^{\prime}\right) e^{+} v_{e}}$, the $\eta-\eta^{\prime}$ mixing angle is determined to be $\phi_{P}=\left(40.2 \pm 1.4_{\text {stat }} \pm 0.5_{\text {syst }}\right)^{\circ}$. From the first measurements of the dynamics of $D_{s}^{+} \rightarrow \eta\left({ }^{\prime}\right) e^{+} v_{e}$ decays, the products of the hadronic form factors $f_{+}^{\eta\left({ }^{\prime}\right)}(0)$ and the CKM matrix element $\left|\mathrm{V}_{c s}\right|$ are extracted with different form factor parameterizations. For the two parameter series expansion, the preliminary results are $f_{\eta}(0)\left|\mathrm{V}_{c s}\right|$ $=0.446 \pm 0.005_{\text {stat }} \pm 0.004_{\text {syst }}$ and $f_{+}^{\eta^{\prime}}(0)\left|\mathrm{V}_{c s}\right|=0.477 \pm 0.049_{\text {stat }} \pm 0.011_{\text {syst }}$. Taking $\left|\mathrm{V}_{c s}\right|$ from the CKMfitter as input, we determine preliminary $f_{+}^{\eta}(0)=0.458 \pm 0.005_{\text {stat }} \pm 0.004_{\text {syst }}$ and $f_{+}^{\eta^{\prime}}(0)=$ $0.490 \pm 0.050_{\text {stat }} \pm 0.011_{\text {syst }}$. Alternatively, using the $f_{+}^{\eta\left({ }^{(\prime)}\right.}(0)$ calculated by light-cone sum rules leads to $\left|\mathrm{V}_{c s}\right|=1.032 \pm 0.012_{\text {stat }} \pm 0.009_{\text {syst }} \pm 0.079_{\text {theo }}$ and $0.917 \pm 0.094_{\text {stat }} \pm 0.021_{\text {syst }} \pm 0.155_{\text {theo }}$, respectively.

\section{Summary}

We present a selection of recent BESIII charm results based on the data sets collected by BESIII detector near the $D \bar{D}$ threshold $(3.773 \mathrm{GeV}), D_{s}^{*} D_{s}$ threshold $(4.178 \mathrm{GeV})$ with integrated luminosities of $2.93 \mathrm{fb}^{-1}$ and $3.19 \mathrm{fb}^{-1}$, respectively. From the leptonic $D_{(s)}$ pure leptonic and semileptonic decays we determined the most precise values for the decay constant $f_{D_{(s)}^{+}}$, the hadronic

form factors $f_{+}^{K(\pi)}(0)$, and the form factor shape $f_{+}^{K(\pi)}\left(q^{2}\right)$ which provide important test to LQCD calculations, and CKM matrix unitary.

\section{References}

[1] M. Ablikim et al. (BESIII Collaboration), Nucl. Instrum. Meth. A 614, 345 (2010).

[2] J. Beringer et al. (Particle Data Group), Phys. Rev. D 86, 010001 (2012).

[3] M. Ablikim et al. (BESIII Collaboration), Phys. Rev. D 89, 051104(R) (2014).

[4] M. Ablikim et al. (BESIII Collaboration), Phys. Rev. D94, 072004 (2016).

[5] M. Ablikim et al. (BESIII Collaboration), Phys. Rev. D 92, 072012 (2015).

[6] M. Ablikim et al. (BESIII Collaboration), Phys. Rev. D 96, 012002 (2017).

[7] H. Na et al. (HPQCD Collaboration), Phys. Rev. D 82, 114506 (2010).

[8] H. Na et al. (HPQCD Collaboration), Phys. Rev. D 84, 114505 (2011).

[9] M. Ablikim et al. (BESIII Collaboration), arXiv:1810.03127 (2018).

[10] M. Ablikim et al. (BESIII Collaboration), Eur. Phys. J.C 76, 369 (2016).

[11] M. Ablikim et al. (BESIII Collaboration), Phys. Rev. Lett. 121, 171803 (2018).

[12] M. Ablikim et al. (BESIII Collaboration), arXiv:1811.02911 (2018). 